\title{
Ansiedade e depressão na adolescência
}

Isabel Brito*

\section{RESUMO}

As perturbações da ansiedade e depressivas são frequentes na adolescência. Provocam situações problemáticas na família, na escola e socialmente. O clínico geral/médico de família, que segue estes doentes ao longo do seu desenvolvimento, está numa posição privilegiada para os avaliar, tratar e referenciar. A confiança que a família e o adolescente depositam nele permite-lhe controlar a evolução e prevenir situações de risco do jovem com o suporte familiar.

Palavras-Chave: Adolescência; Perturbações da Ansiedade e Depressivas; Avaliação; Tratamento; Prevenção; Clínico Geral/ /Médico de Família.

\section{A Adolescência}

A adolescência é um período de transformação, em que múltiplas mudanças físicas, psíquicas, afectivas e sociais têm lugar. No centro destas mudanças está a metamorfose do corpo de criança para o de adulto sexuado. Na criança, o corpo é conhecido, seguro, a relação com os pais é de dependência e protecção que são aceites. Ao entrar na puberdade as alterações hormonais e morfológicas impõem-se, assim como a emergência de novas capacidades de sentir, pensar e agir.

$\mathrm{O}$ adolescente vai enfrentar a necessidade de se redefinir em relação ao seu corpo sexuado, à sua identidade psíquica e ao seu meio, em especial em relação aos seus pais.

A ligação que estabeleceu com os pais, desde os primeiros momentos de vida, que contribuiu para a construção da segurança básica em si e nos outros, a estima de si próprio, a confiança nas suas capacidades, vai ser determinante para enfrentar as profundas mudanças com que se confronta.

$\mathrm{O}$ adolescente coloca a questão: quem sou eu? Segundo Évelyne Kestemberg ${ }^{1}$ «os adolescentes são e consideram-se em função do que são os adultos e da forma como os adultos os consideram». A qualidade da relação que os adultos (pais, professores, amigos), estabelecem com o adolescente, a atenção que lhe dão, são

*Psiquiatra da Infância e Adolescência. decisivas para a edificação da identidade do adolescente.

\section{A ANSIEDADE Introdução}

As profundas transformações vividas pelo adolescente provocam nele um sentimento de inquietação, de estranheza em relação a si próprio. As actividades desportivas ou artísticas que ele tinha anteriormente e de que retirava grande prazer começam a ser postas de lado. Ele sente-se desajeitado, sem controlo sobre o seu corpo e a sua sexualidade. Torna-se crítico, rebela-se à mínima contrariedade, afasta-se.

Agir, é nesta fase, uma forma de descarregar a ansiedade: discutir, ter maus resultados escolares, fugir. ${ }^{2}$

A ansiedade é uma emoção frequente, sinal de alarme perante uma situação que pode constituir uma ameaça.

O adolescente sente-se ameaçado pelas grandes alterações que lhe estão a acontecer: no seu corpo, que não controla, na relação com os pais, em que os conflitos de dependência/autonomia são constantes, nos receios que sente em relação às suas competências sociais, escolares e na relação com os pares.

\section{Identificação dos sintomas}

É frequente apresentarem sintomas físicos, que os fazem entrar em contacto com o Clínico Geral/Médico de Família (CG/MF): dores abdominais, musculares, nos 
membros, nas costas, cefaleias, fadiga. Podem ter uma sudação excessiva, tremer, sentir tonturas e «desmaios».

O CG/MF deve tentar estabelecer uma relação de interesse e empatia com o jovem, para que este consiga expressar as suas emoções e sentir-se mais seguro.

A ansiedade pode interferir com a aprendizagem e com a inserção escolar, comprometer a relação com o grupo de pares, acentuar os conflitos com a família e conduzir ao isolamento do adolescente.

$\mathrm{O}$ adolescente pode desenvolver ataques de pânico ou fobias. Apresentar comportamentos de risco, consumindo álcool e drogas ou ter um comportamento sexual impulsivo, como tentativas para negar os seus medos.

\section{Perturbações da Ansiedade}

A terminologia que usamos neste artigo está de acordo com o DSM-IV-TR (Manual de Diagnóstico e Estatística das Perturbações Mentais (2002). ${ }^{3}$

Pela sua especificidade não abordaremos aqui a perturbação de ansiedade pós-stress traumático.

\section{Ansiedade de Separação}

$\mathrm{O}$ adolescente manifesta receio em se separar dos seus pais e da sua casa. Mostra-se preocupado com a sua saúde e a saúde dos pais. Tem dificuldade em dormir sozinho e tem pesadelos. Apresenta queixas somáticas. Pode recusar-se em ir à escola.

Por vezes manifesta-se após uma situação preocupante como o divórcio dos pais, a doença ou a morte de um familiar próximo. Às vezes um dos pais também apresenta um grande receio com a separação, que comunica ao filho e este evita separar-se. ${ }^{4}$

\section{Perturbação de Ansiedade Generalizada}

Manifesta-se no adolescente por uma preocupação excessiva e mantida em diversas áreas como a escolaridade, as relações sociais, familiares, a saúde, acontecimentos mundiais e desastres naturais.

Pode apresentar queixas somáticas. Tende a ser perfeccionista e geralmente os pais têm uma elevada expectativa em relação às suas realizações mas por outro lado acedem facilmente aos seus pedidos.

Acontece tanto em raparigas como em rapazes. ${ }^{4}$

\section{Perturbação de Pânico}

São episódios agudos de ansiedade intensa acom- panhados de sintomas físicos e emocionais, sem causa aparente.

Mais frequente nas raparigas, inicia-se entre os $15 \mathrm{e}$ os 19 anos. $^{5}$

O adolescente sente um medo intenso, acompanhado por sintomas físicos como uma sudação excessiva, dor no peito, náuseas, tonturas e dificuldade em respirar. Por vezes refere que pensa que vai morrer.

Na tentativa de evitar situações que possam desencadear o ataque de pânico, reduz ou abandona as suas

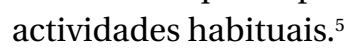

\section{Fobias}

Considera-se uma fobia quando aparece um medo irracional perante uma situação ou objecto específico. ${ }^{5}$

$\mathrm{O}$ adolescente pode apresentar queixas somáticas como gastralgias, cefaleias e náuseas.

$\mathrm{O}$ adolescente pode negar-se a frequentar a escola. Se o afastamento escolar se prolongar, torna-se mais difícil retomar a escola, porque ele sente-se mais afastado dos colegas e com mais receio de não acompanhar as tarefas escolares.

\section{Fobia Social}

A fobia social manifesta-se em contextos sociais como acontecimentos musicais, desportivos e outros. $O$ jovem sente-se incomodado em ambientes não familiares e o desconforto desaparece quando deixa de estar em ambientes sociais. ${ }^{5}$

O adolescente apresenta queixas somáticas, evita os locais que ele pensa que lhe desencadeiam mal estar.

\section{Perturbação Obsessiva-Compulsiva}

$\mathrm{O}$ adolescente manifesta obsessões, pensamentos recorrentes sobre limpeza, contaminação, receio de ter agredido alguém, incluindo os pais, temas sexuais e outros. Estes pensamentos têm correspondência em comportamentos e rituais como frequente lavagem de mãos e outros locais do corpo, permanente revisão e controlo sobre as actividades desenvolvidas, toques repetidos, ordenação, contagem e acumulação de objectos. ${ }^{5}$ O início destes sintomas é geralmente pelos 10 anos.

\section{Diagnóstico Diferencial}

Atenção às situações clínicas que podem provocar estados de ansiedade, sendo necessário um exame mé- 
dico cuidadoso para as excluir. São elas:

- Hipertiroidismo; hipoglicémia; arritmia cardíaca; feocromocitoma; doenças convulsivas; hipoxia e tumores cerebrais. ${ }^{5}$

Há medicamentos e drogas que podem desencadear ansiedade, como:

- Cafeína; nicotina; anti-histamínicos; anti-asmáticos (teofilina); simpaticomim'eticos; esteróides; antipsicóticos; inibidores selectivos da serotonina; estimulantes (incluindo a cocaína); marijuana. ${ }^{5}$

É necessário verificar se o adolescente está a fazer alguma desta medicação ou a tomar outras drogas.

Pode haver um quadro de ansiedade independentemente de tomar ou não estas substâncias. A história clínica é decisiva.

\section{Avaliação e Tratamento}

A perturbação da ansiedade deve ser diagnosticada quando interfere com o funcionamento diário do adolescente e da família. Torna-se necessário avaliar o impacto que está a ter na aprendizagem e na frequência escolar do adolescente, nas relações familiares e com os pares.

O tratamento deve ser planeado envolvendo o adolescente, a família, a escola e por vezes os amigos.

A família deve ser informada que os sintomas da ansiedade são involuntários. Mesmo parecendo que o adolescente pretende "manipular» os que o cercam, não está a fazê-lo por vontade própria. Os pais fazem parte do tratamento. Os pais devem transmitir segurança ao adolescente, não se mostrarem críticos em relação aos seus receios e menos ainda castigá-lo pelos seus comportamentos desajustados. ${ }^{4}$

Como existem quase sempre repercussões do problema na escola, deve ser feito um trabalho em ligação com ela. $\mathrm{O}$ adolescente frequentemente baixa o seu rendimento escolar e não se sente bem na escola; se puder dispor de um professor «tutor» com quem possa falar e o possa ajudar a manter-se na escola, pode ser essencial para o sucesso escolar. Quando o adolescente já não vai à escola, a possibilidade de um professor se deslocar a casa, para o ajudar a organizar o estudo e o manter a par das matérias escolares, para além de o incentivar a retornar à escola, pode ser decisivo para o seu percurso escolar.

Se a ansiedade for moderada e não for inibidora do dia-a-dia do adolescente, o CG/MF poderá explicar e solicitar o suporte dos pais e eventualmente medicá-lo.

Os medicamentos de eleição são os inibidores selectivos da recaptação da serotonina, nomeadamente a sertralina ou a paroxetina. Deve iniciar-se com $1 / 2$ comprimido e ir subindo gradualmente. Os efeitos poderão não ser visíveis antes de 3 a 6 semanas. No caso de uma perturbação obcessiva-compulsiva o efeito poderá não se fazer sentir antes de 10 a 12 semanas. ${ }^{4}$

Uma abordagem psicoterapêutica está indicada.

Quando os sintomas duram há mais de 3 meses e o adolescente não vai à escola há mais de 2 semanas, deve ser enviado, com a família, a um especialista de saúde mental. Será necessária uma abordagem psicoterapêutica e psicofarmacológica.

\section{A DEPRESSÃO}

Introdução

O papel decisivo dos CG/MF na detecção, avaliação e tratamento desta patologia é reconhecido pela Academia Americana de Pediatria, que desenvolveu guidelines, baseadas na evidência científica, para os Cuidados Primários de Saúde sobre a depressão no adolescente. ${ }^{6}$

O papel dos CG/MF é ainda mais relevante pela escassez de especialistas em saúde mental.

Como escasseiam os estudos epidemiológicos na nossa população, iremos socorrer-nos deste trabalho, que, pela nossa prática clínica, julgamos não se distanciar muito da nossa realidade nacional.

A depressão major nos adolescentes é considerada uma séria doença psiquiátrica com uma extensa morbilidade, aguda e crónica, e mortalidade.,

Estudos mostram que, nos cuidados de saúde primários, 2 em 3 adolescentes com depressão não são identificados e não recebem nenhum tratamento. ${ }^{9}$ Dos que são diagnosticados só metade são devidamente tratados. ${ }^{10}$

A perturbação depressiva major (PDM) está descrita na DSM-IV-TR, ${ }^{3}$ que inclui outros tipos de depressão como a perturbação distímica (PD) e a doença bipolar (DB).

\section{OS SÍNDROMES DEPRESSIVOS}

\section{Perturbação distímica}

Há poucos estudos clínicos e ensaios controlados para o tratamento da distimia nos jovens. Segundo a 
American Psychiatric Association, ${ }^{11}$ os tratamentos utilizados na PDM são idênticos aos para a PD.

A PD consiste numa prolongada alteração do humor, durante mais de um ano, com irritabilidade e depressividade, alterações do sono e do apetite, baixa con-

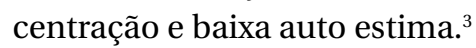

O curso é menos intenso mas mais crónico do que a PDM. Os sintomas não são tão intensos mas causam considerável prejuízo psico-social. ${ }^{12,13}$

Estes sintomas devem ter repercussões negativas em várias áreas da vida do adolescente como o desempenho escolar, nas relações familiares e com os pares.

\section{Doença Bipolar}

É controverso e objecto de debate científico o diagnóstico de doença bipolar na adolescência.

O diagnóstico de doença bipolar deve ser colocado quando o humor do adolescente se mostra expansivo ou eufórico, mesmo explosivo, com manifestações de acentuada irritabilidade por vezes com agressões, agitação psicomotora, falta de sono, falta de atenção e fuga de ideias. ${ }^{8}$

Nos adolescentes o quadro é geralmente misto com o da depressão e pode haver passagem de um para outro quadro.

Segundo a DSM-IV-TR, ${ }^{3}$ a doença bipolar I requer a ocorrência de um episódio maníaco ou misto, com a duração de pelo menos 7 dias. Não é necessário haver episódios depressivos, mas a maioria dos doentes experimentam episódios major ou minor de depressão ao longo da vida.

Segundo alguns estudos a prevalência da doença nos adolescentes é de $1 \% .^{14}$

\section{Factores de risco}

São considerados factores de risco para o eclodir da doença bipolar em adolescentes: (1) episódio depressivo de começo súbito e sintomas psicóticos; (2) história familiar de perturbações afectivas, especialmente doença bipolar; (3) história de mania ou hipomania após tratamento com antidepressivos. ${ }^{15}$

É frequente a comorbilidade com o consumo de substâncias (álcool, cocaína, anfetaminas, opiáceos).

\section{Tratamento}

Considera-se como $1 .^{a}$ linha do tratamento os esta- bilizadores do humor e os antipsicóticos atípicos, devendo juntar-se a psicoterapia e a intervenção junto da família e da escola. ${ }^{16}$

\section{Perturbação Depressiva Major}

A perturbação depressiva major (PDM) na adolescência é considerada uma perturbação sindromática. ${ }^{8}$

Os pais trazem o adolescente ao CG/MF, que, pelo conhecimento que tem da família e a relação já estabelecida com o adolescente, poderá ter uma intervenção de grande valia, pela confiança nele depositada e pela continuidade relacional que proporciona.

A prevalência da depressão está estimada em $4 \%$ a $8 \%$ dos adolescentes, com um rácio de rapaz/rapariga de 1:2. ${ }^{17}$ Aos 18 anos a incidência é de aproximadamente $20 \%{ }^{18}$

O CG/MF pode ser a primeira pessoa a quem o adolescente refere já ter pensado no suicídio $(60 \%$ dos casos referem-no e $30 \%$ já fizeram uma tentativa). ${ }^{19}$

O conhecimento destes casos, que o CG/MF possui, e o seu relacionamento com o jovem permitem-lhe intervir quando a situação clínica surge e controlá-la ao longo dos anos. Mesmo que a situação imponha uma intervenção especializada, o CG/MF não deve "abandonar" o caso, deve manter a relação com o jovem e a vigilância sobre a sua evolução.

A recorrência é frequente, cerca de $20 \%$ a $60 \%$ nos primeiros 2 anos após a remissão e $70 \%$ após 5 anos de remissão. ${ }^{20,21}$ Estas recorrências continuam na vida adulta. Os adolescentes com depressão estão em alto risco de desenvolverem dependência de drogas, doenças físicas, dificuldades no trabalho académico, na inserção social e terem problemas com a lei. ${ }^{8}$

A PDM manifesta-se de forma polifacetada, por uma alteração persistente do humor, irritabilidade, comportamento de oposição, diminuição do desempenho escolar, desistência de actividades desportivas ou culturais, até então apreciadas, afastamento dos amigos e queixas somáticas, como cefaleias, gastralgias, insónia, falta de apetite ou fadiga. ${ }^{3}$

\section{Factores de Risco}

Vários são os factores de risco para a depressão no adolescente, que o CG deve valorizar na história pessoal e familiar: (1) depressão, (2) doença bipolar, (3) comportamentos suicidas, (4) abuso de drogas, (5) ou- 
tras doenças psiquiátricas, (6) transtornos a nível psico-social como crises na família, negligência, abuso físico ou sexual ou outra circunstância traumática. ${ }^{22,23}$

O risco de comportamento suicida aumenta se houver história de tentativas de suicídio, comorbilidade com outras perturbações psiquiátricas, abuso de drogas, impulsividade e agressão, exposição a acontecimentos violentos como abuso físico ou sexual, acesso a armas letais e história familiar de suicídio. ${ }^{24}$

O factor considerado o mais predisponente de PDM é a carga familiar desta patologia. ${ }^{25}$

A auto-mutilação não tem como finalidade acabar com a vida, mas representa uma forma de circunscrever a dor psíquica a uma dor física, localizada e passageira. Como o êxito é momentâneo, tende a repetir-se e pode evoluir para tentativas de suicídio.

\section{Avaliação}

A avaliação deve ser feita quer com o adolescente quer com a família, em separado. ${ }^{26}$

O envolvimento da família é crucial em todas as fases da intervenção.

A comorbilidade deve ser avaliada: abuso de drogas, perturbações ansiosas, doença bipolar, doença física, abuso físico, sexual ou outro trauma. ${ }^{27}$

A possibilidade de uma gravidez deve ser equacionada.

As alterações no funcionamento escolar, familiar e com os amigos, devem ser estimadas. ${ }^{28}$

\section{Diagnóstico Diferencial}

Algumas doenças médicas podem causar sintomas idênticos aos da depressão, como o hipotiroidismo, a mononucleose infecciosa, a anemia, as neoplasias e as doenças auto-imunes.

Também alguns medicamentos, como os estimulantes, os corticóides e os contraceptivos, podem desencadear sintomas idênticos aos depressivos. ${ }^{27}$

\section{Tratamento}

Uma das primeiras atitudes terapêuticas que o CG/MF deve assumir é a partilha de conhecimentos com o jovem e a família, sobre as causas e sintomas da depressão, os danos que pode causar e quais as expectativas em relação ao tratamento. ${ }^{29}$

O plano de tratamento deve ser discutido e acorda- do entre todos. Deve ser sempre plurimodal, incluindo psicoterapia, medicação e o envolvimento da família.

O tratamento tem como finalidade a resolução dos sintomas depressivos e melhoria do funcionamento intra e interpessoal, escolar, familiar e social do adolescente.

A psicoterapia é uma abordagem considerada de $1 .^{\mathrm{a}}$ linha.

A medicação preconizada é com anti-depressivos que sejam inibidores selectivos da recaptação da serotonina, como a Sertralina, o Escitalopram, Paroxetina, a Fluvoxamina e a Fluoxetina. As doses iniciais devem ser baixas e irem aumentando progressivamente. ${ }^{27}$

Refere-se o que a GLAD-PC ${ }^{27}$ preconiza como seguimento desta patologia, que releva a sua gravidade na vivência actual e futura do jovem, apesar do reconhecimento da dificuldade destes procedimentos pelos nossos Cuidados de Saúde Primários.

A GLAD-PC recomenda a observação do adolescente e da família 1 semana após o início do tratamento e durante 4 semanas seguidas. Depois de $2 / 2$ semanas durante 4 semanas. A seguir às 12 semanas e assim sucessivamente. $O$ tratamento deve durar 1 ano para evitar as recaídas. O maior risco de recaída é nas 8 a 12 semanas após descontinuar a medicação. Por isso a medicação deve ser mantida por 6 a 12 meses após a completa resolução dos sintomas. O contacto telefónico deve ser assegurado no intervalo das consultas. Todos os doentes devem ser monitorizados mensalmente durante 6 a 12 meses após a total resolução dos sintomas. ${ }^{27}$

A intervenção deve considerar que a depressão é uma situação recorrente.

Se o episódio depressivo é uma recorrência, o clínico deverá monitorizá-la por um período de 2 anos e manter a medicação por 2 anos após a resolução dos sintomas.

Se a resposta ao tratamento for parcial, é necessário considerar se está a haver pouca adesão ao tratamento, comorbilidade, ou se existem outros conflitos na família ou mesmo situações de abusos.

O adolescente e a família devem ser informados sobre os limites da confidencialidade, no caso do adolescente estar em risco iminente de fazer algum mal, a si ou aos outros.

O doente deve ser referenciado a um especialista de saúde mental, quando o tratamento não está a surtir 
efeito, há comorbilidade com o uso de drogas, psicose ou ideação suicida.

Quando o doente é referenciado a ligação com o CG/MF deve ser mantida. Vem sendo demonstrado à evidência que os cuidados partilhados são os de maior efectividade. ${ }^{30}$

A melhoria dos cuidados passa pela mudança dos sistema de saúde, que deverão providenciar conhecimentos e treino em saúde mental aos profissionais dos Cuidados de Saúde Primários com modelos de colaboração entre os diversos profissionais.

\section{Prevenção}

O CG/MF, ao observar um adolescente cuja família tem uma história de depressão, ou que já teve um episódio depressivo ou ainda se apresenta sinais ligeiros de depressão, deve ficar atento à sua evolução, que poderá ser para um quadro depressivo. ${ }^{31}$

O tratamento de mães com depressão mostrou que os filhos apresentavam menos problemas psiquiátricos, ou, os que existiam, tinham uma maior taxa de remissão. 32,33

A identificação e o tratamento precoce de mães deprimidas são decisivos para o adequado desenvolvimento dos seus filhos. ${ }^{34}$

A PD está associada a um maior risco de PDM, ${ }^{12}$ indicando que deve ser tratada quando surge.

Há evidência que a perturbação ansiosa é percursora de depressão $0^{35,36}$ e que o seu tratamento reduz o início e as recorrências da depressão.

A prevenção da depressão releva a abordagem dos factores de risco nela implicados.

É importante informar os jovens, os pais e os profissionais, que com eles se relacionam, sobre os sinais da depressão e as medidas a tomar.

\section{Conclusão}

Um desafio é lançado aos profissionais dos Cuidados de Saúde Primários, para se ocuparem não só da saúde física como da saúde mental dos seus utentes. É algo a que já se dedicam, mas necessitam de uma maior partilha de conhecimentos e práticas com os profissionais da área da saúde mental. Neste caso, para melhor ajudarem os seus jovens pacientes a apoderarem-se das suas competências e a adquirirem um papel activo na procura do seu lugar na vida.

\section{REFERÊNCIAS BIBLIOGRÁFICAS}

1. Kestemberg E. L'adolescence à vif. Paris: Presses Universitaires de France; 1999.

2. Jeammet P. La dépression chez l'adolescent. In: Traité de psychiatrie de l'enfant et de l'adolescent, tome II. Paris: Presses Universitaires de France; 1985. p. 305-25.

3. DSM-IV-TR Manual de Diagnóstico e Estatística das Perturbações Mentais. Lisboa: Ed. Climepsi; 2002.

4. Kaye D, Montgomery M, Munson S. Child and Adolescent Mental Health. Philadelphia: Lippincott Williams \& Wilkins; 2002.

5. American Academy of Child and Adolescent Psychiatry. Practice parameter for the assessment and treatment of children and adolescent with anxiety disorders. J Am Acad Child Adolesc Psychiatry 2007 Feb; 46 (2): 267-83.

6. Zuckerbrot RA; Cheung AH, Jensen PS, Stein RE, Laraque D; GLAD-PC Steering Group. Guidelines for adolescent depression in primary care (GLAD-PC): I. identification, assessment, and initial management. Pediatrics 2007 Nov; 120 (5): e1299-e1312.

7. Fleming JE, Offord DR, Boyle MH. Prevalence of childhood and adolescent depression in the community. Ontario Child Health Study. Br J Psychiatry 1989 Nov; 155: 647-54.

8. American Academy of Child and Adolescent Psychiatry. Practice parameter for the assessment and treatment of children and adolescent with depressive disorders. J Am Acad Child Adolesc Psychiaty 2007 Nov; 46 (11): 1503-26.

9. Burns BJ, Costello EJ, Angold A, Tweed D, Stangl D, Farmer EM, et al. Children's mental health service use across service sectors. Health Aff (Millwood) 1995 Fall; 14 (3): 147-59.

10. Kessler RC, Avenevoli S, Ries Merikangas K. Mood disorders in children and adolescents: an epidemiologic perspective. Biol Psychiatry 2001 Jun 15; 49 (12): 1002-14.

11. American Psychiatric Association. Practice guidelines for the treatment of patients with major depressive disorder (revision). Am J Psychiatry 2000 Apr; 157 (4 Suppl): 1-45.

12. Kovacs M, Akiskal HS, Gatsonis C, Parrone PL. Childhood-onset dysthymic disorder: clinical features and prospective naturalistic outcome. Arch Gen Psychiatry 1994 May; 51 (5): 365-74.

13. Masi G, Favilla L, Mucci M, Poli P, Romano R. Depressive symptoms in children and adolescents with dysthymic disorder. Psychopathology 2001 Jan; 34 (1): 29-35.

14. Geller B, Luby J. Child and adolescent bipolar disorder: a review of the past 10 years. J Am Acad Child Adolesc Psychiatry 1997 Sep; 36 (9): 1168-76.

15. Strober M, Carlson G. Bipolar illness in adolescents with major depression: clinical, genetic and psychopharmacologic predictors in a 34 year prospective follow-up investigation. Arch Gen Psychiatry 1982 May; 39 (5): 549-55.

16. McClellan J, Kowatch R, Findling RL;Work Group on Quality Issues. Practice parameter for the assessment and treatment of children and adolescents with bipolar disorders. J Am Acad Child Adolesc Psychiatry 2007 Jan; 46 (1): 107-25.

17. Birmaher B, Ryan ND, Williamson DE, Brent DA, Kaaufman J, Dahl RE, et al. Childhood and adolescent depression: a review of the past ten years. Part I. J Am Acad Child Adolesc Psychiatry 1996 Nov; 35 (11): 
1427-39.

18. Lewinsohn PM, Rohde P, Seeley JR. Major depressive disorder in older adolescents: prevalence, risk factors, and clinical implications. Clin Psychol Rev 1998 Nov; 18 (7): 765-94.

19. American Academy of Child and Adolescent Psychiatry. Practice parameter for the assessment and treatment of children and adolescents with suicidal behavior. J Am Acad Child Adolesc Psychiatry 2001 Jul; 40 (7 Suppl): 24S-51S.

20. Birmaher B, Arbelaez C, Brent D. Course and outcome of child and adolescent major depressive disorder. Child Adolesc Psychiatr Clin N Am 2002 Jul; 11 (3): 619-37.

21. Costello EJ, Pine DS, Hammen C, March JS, Plotsky PM, Weissman MM, et al. Development and natural history of mood disorders. Biol Psychiatry 2002 Sep 15; 52 (6): 529-42.

22. Jensen PS. Closing the evidence-based treatment gap for children's mental health services: what we know versus what we do. Rep Emotional Behav Disord Youth 2002 Spring; 2 (2): 43-7.

23. Katon W, Rutter C, Ludman EJ, Von Korff M, Lin E, Simon G, et al. A randomized trial of relapse prevention of depression in primary care. Arch Gen Psychiatry 2001 Mar; 58 (3): 241-7.

24. Beautrais AL. Risk factors for suicide and attempted suicide among young people. Aust N Z J Psychiatry 2000 Jun; 34 (3): 420-36.

25. Weissman MM, Wickramaratne P, Nomura Y, Warner V, Vderdeli H, Pilowsky DJ, et al. Families at high and low risk for depression: a 3-generation study. Arch Gen Psychiatry 2005 Jan; 62 (1): 29-36.

26. Jensen PS, Rubio-Stipec M, Canino G, Bird HR, Dulcan MK, SchwabStone ME, et al. Parent and child contributions to diagnosis of mental disorder: are both informants always necessary? J Am Acad Child Adolesc Psychiatry 1999 Dec; 38 (12): 1569-79.

27. Cheung AH, Zuckerbrot RA, Jensen PS, Ghalib K, Laraque D, Stein RE; GLAD-PC Steering Group. Guidelines for Adolescent Depression in Primary Care (GLAD-PC): II. Treatment and ongoing management. Pediatrics 2007 Nov; 120 (5): e 1313-26.

28. Curry J, Rohde P, Simons A, Silva S, Vitiello B, Kratochvil C, et al. Predictors and moderators of acute outcome in the Treatment for Adolescents With Depression Study (TADS). J Am Acad Child Adolesc Psy- chiatry 2006 Dec; 45 (12): 1427-39.

29. Stein RE, Zitner LE, Jensen PS. Interventions for adolescent depression in primary care. Pediatrics 2006 Aug; 118 (2): 669-82.

30. Lang AJ, Norman GJ, Casmar PV. A randomized trial of a brief mental health intervention for primary care patients. J Consult Clin Psychol 2006 Dec; 74 (6): 1173-9.

31. Beardslee WR, Versage EM, Van de Velde P, Swatling S, Hoke H. Preventing depression in children through resiliency promotion: the prevention intervention project. In: McMahon RJ, DeV Peters R, editors. The Effects of Parental Dysfunction on Children. New York: Kluwer Academic/Plenum; 2002. p. 71-86.

32. Weissman MM, Pilowsky DJ, Wickramaratne PJ, Talati A, Wisniewski SR, Fava $M$, et al. Remissions in maternal depression and child psychopathology: a STAR*D-child report. JAMA 2006 Mar 22; 295 (12): 1389-98.

33. Weissman MM, Wickramaratne P, Nomura Y, Warner V, Pilowsky D, Verdeli H. Offspring of depressed parents: 20 years later. Am J Psychiatry 2006 Jun; 163 (6): 1001-8.

34. Brito I. A saúde mental na gravidez e primeira infância. Rev Port Clin Geral 2009 Set-Out; 25 (5): 600-4.

35. Kovacs M, Gatsonis C, Paulauskas SL, Richards C. Depressive disorders in childhood: IV. A longitudinal study of comorbidity with and risk for anxiety disorders. Arch Gen Psychiatry 1989 Sep; 46 (9): 776-82.

36. Pine DS, Cohen P, Gurley D, Brook J, Ma Y. The risk for early-adulthood anxiety and depressive disorders in adolescents with anxiety and depressive disorders. Arch Gen Psychiatry 1998 Jan: 55 (1): 56-64.

\section{CONFLITOS DE INTERESSE}

A autora não possui qualquer conflito de interesses.

\author{
ENDEREÇO PARA CORRESPONDÊNCIA \\ Isabel Brito \\ E-mail: isa.brito@yahoo.com
}

Recebido em 19/02/2011

Aceite para publicação em 17/04/2011

\section{ABSTRACT}

\section{ANXIETY AND DEPRESSION DISORDERS IN ADOLESCENTS}

Anxiety and depressive disorders occur commonly in adolescents. They are a significant cause of familiar, school and social dysfunction and distress. As general practitioner (GP) follows patients through developmental stages, he is well positioned to assess, treat and refer these patients. The family and the adolescent trust in the GP who allow him to monitor evolution and prevent risk situations with parents engagement.

Keywords: Adolescent; Anxiety Disorders, Depressive Disorder; Diagnosis; Drug Therapy; Prevention and Control; General Practitioners. 